\title{
Disease Surveillance Strategies in Swine
}

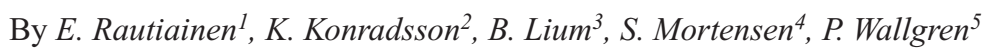

${ }^{1}$ National Veterinary and Food Research Institute, Regional Laboratory in Seinäjoki, P.O. Box 198, FIN-60101 Seinäjoki, Finland, ${ }^{2}$ Tilraunastod Haskola Islands, Keldum v/ Vesturlandsveg, IL-110 Reykjavik, Iceland, ${ }^{3}$ Norwegian Pig Health Service, Veterinærinstituttet, Boks 8156 dep., N-0033 Oslo, Norway, ${ }^{4}$ Danish Slaughterhouses, Axelborg, Axeltorv 3, DK-1609 Copenhagen V, Denmark, ${ }^{5}$ National Veterinary Institute, P.O. Box 7073, SE-75007 Uppsala, Sweden.

\section{Pig production systems in the Nordic coun- tries}

The volume and the structure of pig production in different Nordic countries is described in table 1 . The volume of the production is clearly largest in Denmark countries, and $80 \%$ of the Danish production is exported. The infrastructure in Denmark and Sweden is similar with respect to the proportional number of nucleus and multiplying herds. Finland and Norway still have a relatively high number of nucleus herds. The pig population in Iceland is minor, excluding nucleus or multiplying herds. There is no export of pigs or pig products from Iceland. Therefore no official disease surveillance programs have existed until today. However, import of pigs to Iceland has been very strictly ruled. The latest imports took place in 1994 and
1996 from Norway and Finland, respectively. It is believed that the health status of Icelandic pigs is similar to that of Norway and Finland. Random sampling of pigs from the majority of herds during 1993-1999 supports that suggestion with respect to the epidemic pig diseases.

\section{National surveillance systems of List $A$ and List B diseases (OIE)}

Today, all Nordic countries are considered free from Aujeszky's disease (AD), transmissible gastroenteritis (TGE), classical swine fever (CSF), and swine vesicular disease (SVD). The aim of the surveillance for these diseases is to document freedom from disease within the national pig populations. The numbers of samples analysed annually for these diseases, and the strategies for collection are shown in table 2.

Table 1. Approximate numbers of different kinds of pig herds, total number of sows and annual number of pigs slaughtered in Nordic countries in 1998.

\begin{tabular}{|c|c|c|c|c|c|}
\hline & Denmark & Sweden & Finland & Norway & Iceland \\
\hline Nucleus herds & 60 & 40 & 150 & $110^{\mathrm{b}}$ & - \\
\hline Multiplying herds & 230 & 150 & 20 & 85 & - \\
\hline \multicolumn{6}{|l|}{ Conventional herds } \\
\hline - farrowing & 2.800 & 5.100 & 2.300 & 500 & - \\
\hline - farrowing-to-finishing & 5.500 & 760 & 1.400 & 3.400 & 54 \\
\hline - finishing & 10.000 & 5.800 & 1.800 & 2.000 & - \\
\hline - sow pool systems & - & 30 & 8 & 1 & - \\
\hline Total no. of sows (thousands) & 1.000 & 274 & $185^{\mathrm{a}}$ & 65 & 4 \\
\hline \multicolumn{6}{|l|}{ Annual no. of pigs } \\
\hline Slaughtered (mill.) & 21 & 3.9 & 2.2 & 1.3 & 0.065 \\
\hline
\end{tabular}

${ }^{a}$ Gilts older than 200 days of age included

${ }^{\mathrm{b}}$ Aspirant herds included 
Table 2. Approximate numbers of annual surveillane samples analysed for serum antibodies to the causative agents of Aujeszky's disease (AD), transmissble gastroenteritis (TGE), classical swine fever (CSF), and swine vesicular disease (SVD) in Nordic countries. In 1998, these countries were considered free of all these disease.

\begin{tabular}{lrlll}
\hline & Denmark & Sweden & Finland & Norway \\
\hline AD & $30.000^{\mathrm{a}}$ & $5.000^{\mathrm{b}}$ & $9.800^{\mathrm{c}}$ & $4.700^{\mathrm{d}}$ \\
TGE & $1.500^{\mathrm{e}}$ & 3.000 & 9.800 & 4.700 \\
$\mathrm{CSF}$ & 30.000 & 3.000 & 2.900 & - \\
SVD & 3.000 & 3.000 & 2.600 & - \\
\hline
\end{tabular}

a A systematic sample comprising $10 \%$ of boars and $5 \%$ of sows slaughtered or exported for slaughter; in border areas all boars and $10 \%$ of sows intended for slaughter are sampled.

b A systematic sample from slaughtered sows and fatteners.

c The sample comprises all boars slaughtered annually, all sows slaughtered during April and May (corresponding to $10 \%$ of total sows slaughtered), and a systematic sample $(n=3000)$ from fatteners.

d All elite breeding and multiplying herds $(\mathrm{n}=200)$, and a stratified random selection ( $\mathrm{n}=290$; fattening herds Underrepresented) of the rest of the pig herds are tested; samples are collected first and foremost from sows.

e Samples from exported live animals.

AD has never been reported in Finland, Norway and Iceland. Denmark and Sweden have eradicated the disease in 1991 and 1996, respectively. The AD free status has been confirmed by the EU commission and by the EFTA's Supervisory Authority (Norway). An annual surveillance for the disease are required in each country.

TGE has never been reported in Denmark, Sweden, Norway or Iceland. In Finland, two cases of TGE of foreign origin were found in 1981. The infection was immediately eradicated. Finland and Norway have been granted additional guarantees relating to TGE by the EFTA's Supervisory Authority. These decisions require an annual surveillance of the disease and reporting of the results.

The surveillance for CSF and SVD in Denmark, Sweden, and Finland is based on deci- sions of the EU commission.

\section{Salmonella infections in pig Denmark}

Aim of the surveillance and organisation

The aim of the Danish Salmonella surveillance program is to reduce the prevalence of Salmonella in slaughter pig herds and pork. Target population includes all herds producing more than 100 slaughter pigs per year. The number of such herds is about 16000 . A permanent program, launched by the Danish Ministry of Agriculture, was established in 1995. The compulsory program is administered by the Danish Meat and Bacon Council.

In addition to surveillance and control of Salmonella in breeding and multiplying herds and in finishing herds, the program involves control of Salmonella in feed stuffs, and control measures at the abattoirs. These parts of the program are beyond the scope of this paper.

\section{Sampling}

Meat juice samples are collected at random from each herd participating in the program. Between eight and sixty meat samples are collected from each herd every quarter of the year. The sample size is predetermined by the number of pigs delivered for slaughter

The system is based on the identification of each herd by a unique code (the Central Herd Registration; CHR) that identifies a herd as the smallest epidemiological unit of trace-back within a given geographical location. Each year, all farmers are obliged by law to inform the CHR register about ownership, herd type and number of pigs. The per-herd systematic sampling frequency is based on a monthly update from Central Zoonoses Register (ZOOR), which is part of the CHR register. ZOOR receives monthly information of the number of pigs slaughtered per herd, and of the actual samples obtained. Around 800000 meat juice samples are examined annually at the Danish 
Veterinary Laboratory.

Tests and testing procedures

An indirect enzyme-linked immunosorbent assay (ELISA) based on a combination of the lipopolysaccharide (LPS) antigens O:1, 4, 5, 6, 7, and 12 is used. This ELISA (Exiqon, Denmark) is presumed to be able to detect O-antigens from at least $93 \%$ of all serovars presently occurring in Danish pigs. In experimental studies using $S$. typhimurium and $S$. infantis, the sensitivities have been $>95 \%$ and the specificities $100 \%$, respectively. Using results obtained with serum as the gold standard, relative sensitivities from 0.81 to 0.89 have been demonstrated when meat juice samples have been analysed. The results of the examination are summarized monthly for individual herds. Based on the proportions of sero-reactors during the last three months, the herds are assigned to one of three levels. Level 1 herds have no or very few sero-reactors. Level 2 herds have a relatively high proportion of sero-reactors (from $>50 \%$ in the smallest to $>10 \%$ in the largest herds), whereas level 3 herds have an unacceptably high proportion of seroreactors $(>50 \%$ for most herd sizes). Both the herd owners and the slaughter companies are informed monthly about the Salmonella level of the herds.

\section{Consequences in the case of assignment to level} 2 or 3

Herds assigned to levels 2 and 3 are requested by the slaughter company to seek advice on how to reduce the prevalence of Salmonella. A sufficient number of faecal samples must be collected and analyzed in order to clarify the distribution of Salmonella in the herd. Based on the results obtained, the herd owner, a veterinarian, and a pig consultant elaborate a herdspecific intervention plan. The recommended measures of the control of disease spread are implementation of all-in/all-out practices in growing and finishing barns, cleaning and dis- infection between batches, and strict control over the risks to introduce external sources of Salmonella.

The slaughter companies collect a penalty per pig delivered if the plan is not elaborated and received by the slaughter companies within 35 days. If a herd remains in level 2 or 3 for more than 6 months, the owner must again seek advice on how to reduce the Salmonella prevalence in the herd. In addition, a slaughtering fee is charged to all herds assigned to level 3 for more than 6 months. The fee would be collected until the herd is reassigned to level 1 or 2. All changes in levels are approved by the Danish Veterinary Service. The slaughter companies update ZOOR about compliance regarding advisory visits.

\section{Current status}

The prevalence of seropositive meat juice samples varied between 4\% and 7\% from 1995 to the end of 1997. A significant decrease was observed from October 1997, to June 1998, reaching a minimum at $2.3 \%$. Since then, the number of seropositive meat juice samples has remained below $3 \%$. The percentage of herds designated at level 2 and level 3 have ranged from $2.4 \%$ to $4.3 \%$ and $1.1 \%$ to $2.3 \%$, respectively. From February 1998 the number of level 2 herds has remained below 3\% and the number of level 3 herds has varied between $1.2 \%$ and $1.8 \%$.

From June 1995 to August 1998, about 25\% of the herds (3955 out of 16000) have been required to seek advice on how to reduce the Salmonella prevalence in the herd. In 1747 herds ( $44 \%$ of the total surveyed), the high prevalence of Salmonella lasted more than six months. In 233 herds (5,9\%), the Salmonella has been so persistent that five to seven requirements to seek advice have been required. In order to reduce the number of herds with chronic Salmonella problems, teams of "second opin- 
ion" advisers have been established. Severe penalties are placed on herds that do not follow the advice of these teams.

\section{Sweden, Finland and Norway}

Aims of the surveillance and organisation

Sweden, Finland, and Norway have a similar national Salmonella control program. The aims of those programs are to maintain a low prevalence of Salmonella in pigs and pig products, to provide reliable recording for the Salmonella status in the pig populations and to prevent increased occurrence of Salmonella. Salmonella infections in pigs have always been notifiable in these countries. The national programs were introduced in 1995.

In addition to surveillance of Salmonella in intestinal lymph nodes at abattoirs, the programs involve surveillance for Salmonella by faecal samples in nucleus, multiplying herds (Sweden and Norway) as well as in sow pool herds (Sweden). In Finland, all boars intended for semen collection centres are individually examined for Salmonella.

In the national programs, Salmonella is also controlled in cattle and poultry, and in fresh meat of cattle and swine. Moreover, great efforts are made within feed industries aiming not to introduce Salmonella to the animals by feed. In addition, both in Sweden and Finland there are voluntary slaughter company activities aiming at certifying herds free from Salmonella. However, these parts of Salmonella control are beyond the scope of this review.

The affiliation to the surveillance program is compulsory in each country. In Sweden and Finland, the costs of sampling and analyses are paid by the industry and the pig producers. Confirmatory analyses are paid by the Animal Health Authorities. In Norway, all costs of the program are paid by the Norwegian Animal Health Authority. Follow-up of the control program is carried out by the national veterinary institutes in each country.

Samples from live animals are collected by the local official veterinarians. At the slaughterhouses, samples are collected by the meat inspection team under the control of an official veterinarian. The analyses are performed by the municipal food control laboratories and by the slaughterhouse laboratories. All Salmonella isolates are confirmed and typed by the national reference laboratories. Animal Health Authorities (provincial veterinarians and local official veterinarians) are responsible for the measures taken at Salmonella findings.

\section{Sampling of faecal samples from pig herds}

In Sweden, faecal samples are collected from 50 individual sows. At laboratory 10 cultivations are made, each representing a pool of 5 samples. In Finland, two pooled faecal samples are collected from 10 pens with animals aged 5 weeks to 6 months. In Norway, pooled faecal samples (representing at least 5 individuals in each pen) are collected from 3 to 20 pens with animals aged 2-6 months.

\section{Sampling of intestinal lymph nodes at abattoirs} The total number of individuals tested in each country is calculated to be sufficient to detect Salmonella at a prevalence level of $0.1 \%$ in the whole pig population with a confidence interval of $95 \%$. The targeted annual sample size in Sweden and Finland is 6000, and in Norway 3000. In Sweden, the daily sample size is equal at all major slaughterhouses $(n=11)$. In Finland and Norway, sampling is stratified to all slaughterhouses and systematically distributed evenly over the year. The numbers of pigs to be sampled at each slaughterhouse is pre-determined by the number of pigs slaughtered during the previous year. The number of slaughterhouses comprising about $99 \%$ of pigs slaughtered is 12 and 30 in Finland and Norway, respectively. All other establishments are treated as one slaugh- 
terhouse. The minimum number of samples collected annually in each slaughterhouse is 59 . In each country, five lymph nodes are always pooled before the bacteriological examination.

\section{Tests and testing procedures}

The nucleus and multiplying herds are tested once a year. The sow pool herds (Sweden) are tested twice a year. The surveillance at slaughterhouses is continuous. Bacteriological examinations are performed according to the Nordic Committee on Food Analysis (NMKL) Method No. 71 (1991). In Finland, also ISO-method 6579 (1990) can be used. All positive samples are confirmed and serotyped by national reference laboratories.

\section{Consequences of a positive test result}

All Salmonella isolations lead to restrictions at the farm imposed by the official veterinarian, aiming $t$ to prevent transmission to other herds and to prevent contamination of food products. Individual faecal samples are taken from sows and boars and from pens with growing and finishing pigs. If Salmonella is demonstrated, actions are taken to eliminate the infection from the herd. In addition, an investigation aiming at finding the source of the infection or contamination is initiated. Special hygienic measures are undertaken at the abattoirs when animals from a Salmonella infected herd are sent to slaughter.

The restrictions of a previously Salmonella infected herd may be removed when individual faecal samples from all sows and boars, and pooled samples from all pens with growing and finishing pigs are tested twice with an interval of at least 30 days and no Salmonella is detected. In herds with fattening pigs the restrictions are released when all animals have been slaughtered and the premises have been disinfected according to the instructions of the official veterinarian.

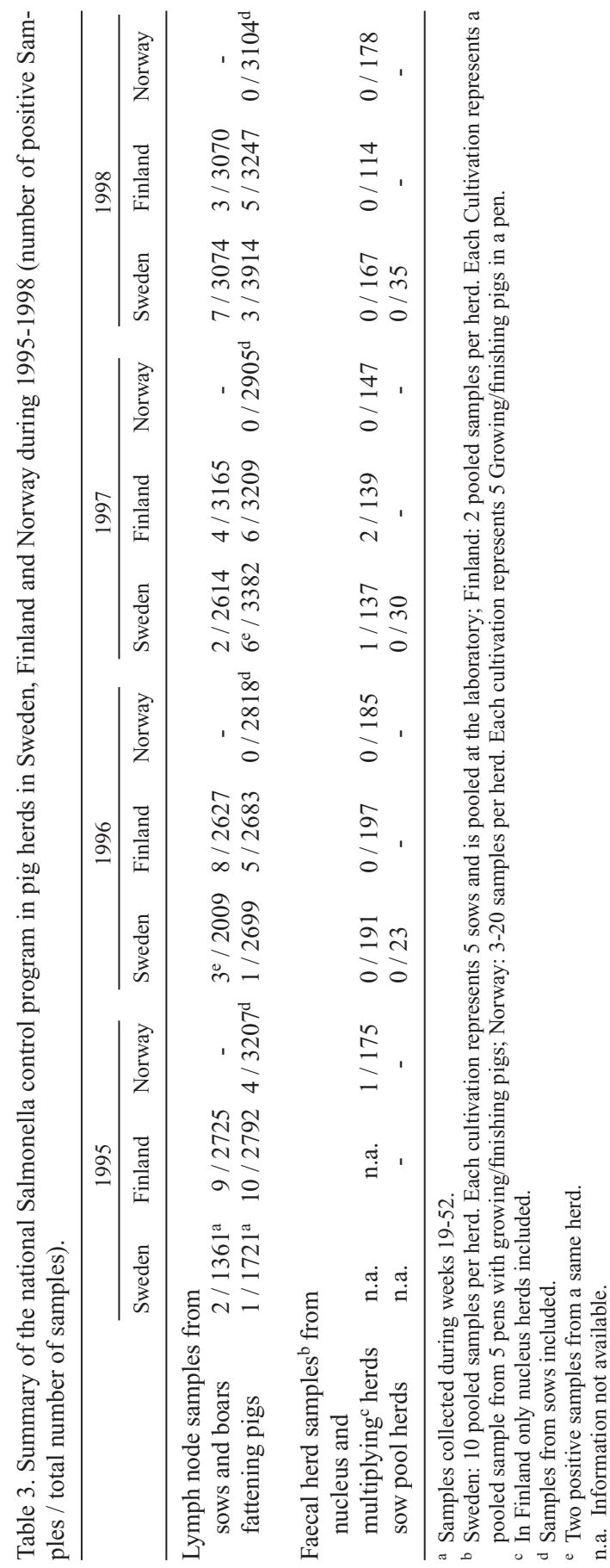

Acta vet. scand. Suppl. 94 - 2001 


\section{Current status}

Results of the continuous surveillance in 19951998 are shown in table 3. In Sweden, Salmonella has been detected in $0.04 \%-0.18 \%$ of the lymph node samples annually. In spite of confirmatory analyses, Salmonella was only demonstratedby faecal samples in one herd during 1996. During 1997, Salmonella was demonstrated in faecal samples of one multiplying herd.

In Finland, Salmonella has been detected in $0.10 \%-0.34 \%$ of lymph node samples annually. Salmonella has not been re-isolated in faecal samples of such herds. In 1997, Salmonella was found in faecal samples of 2 nucleus herds $(S$. enteritidis FT 8; S. typhimurium FT 1). In the latter herd, Salmonella could also be re-isolated once (S. typhimurium var. Copenhagen). Both herds became clean very soon.

In Norway, Salmonella was not detected in any lymph node samples or faecal samples during 1996-1998. In 1995, Salmonella was found in faecal samples of one multiplying herd (S. agona) and in 4 lymph node samples $(0.12 \%)$. Salmonella was not demonstrated by cultivation in these herds.

\section{Iceland}

In Iceland, 447 faecal samples were taken from 29 herds during 1991-1995. One herd has been found positive for S. typhimurium and the herd was slaughtered out.

\section{Porcine Reproductive and Respiratory Syndrome (PRRS) \\ Denmark}

Aim of the monitoring and organisation Isolation of PRRS-virus is notifiable in Denmark. Notification is a prerequisite for export of meat from non-outbreak herds to a few markets.
Serological monitoring of herd infection status is compulsory in nucleus and multiplying herds $(n=360)$, and voluntary in production herds. The aim of the monitoring is to assist the trade of live pigs between herds of similar infection status. The secondary purposes include monitoring of national and regional levels of PRRSvirus infection. This information may be used to make decisions on national PRRS policies, regional PRRS policies by farmer groups, and to make decisions related to cost-benefit of eradication procedures at the farm level. A PRRS-database was developed in February 1996. The database is administered by the Danish Meat and Bacon Council, which also pays for administration and distribution of information. Farmers pay for sample collection and diagnostic services.

The monitoring of nucleus and multiplying herds is based on serum samples collected from the herds. Samples are analysed for antibodies at the Danish Veterinary Institute for Virus Research. Monitoring of production herds is based on serum samples or on meat juice samples collected at the slaughterhouses. The latter samples are analysed for antibodies to PRRS at the Danish Veterinary Laboratory.

\section{Sampling}

From all breeding and multiplying herds, 10 serum samples are collected monthly from randomly chosen growing and finishing pigs. From production herds, 20 serum samples are collected annually. Alternatively, 40 meat juice samples are collected systematically throughout the year. A unique herd code (Central Herd Registration code) provides basic herd information for any diagnostic investigation.

\section{Tests and testing procedures}

The serum samples from nucleus and multiplying herds are analysed monthly for presence of antibodies to PRRS by a blocking-ELISA 
aimed at detecting antibodies to both European and US sub-types of PRRS virus. Meat juice samples from production herds are analysed for PRRS-antibodies by an indirect ELISA. A positive result indicate that the herd has been infected by PRRS-virus.

\section{Consequences of a positive test result}

A "PRRS suspect" herd status is assigned when the positive predictive value of meat juice test results exceeds the values corresponding to those obtained in 2 positive samples out of 10 . Actions following assignment of "PRRS suspect" herd status include notification of the farmer, the herd veterinarian and trade organisations, and collection of 10 additional meat juice samples intended for clarification of PRRS herd status. If the herd is infected the farmer must not sell pigs to PRRS herds.

\section{Certification}

The PRRS database does not certify the infection status of the herds in legal terms. The farmer himself is responsible for providing information of the true infection status of his herd in relation to his customers. The SPF company certify the PRRS status of the herds, and if required it demands herds to participate in the PRRS database.

\section{Record keeping}

The Central Herd Register in the Ministry of Food, Agriculture and Fisheries provide basic herd information for the PRRS database. By his signature, the individual farmer represents his herd in the database. He authorises that diagnostic results from the laboratories are forwarded to the database, and that trade companies, the herd veterinarian and the swine specialists gain access to the PRRS herd infection status. The diagnostic results are kept for indefinitely unless the farmer requests removal of his herd from the database.

\section{Current status}

The estimated PRRS prevalence was $25 \%$ in nucleus and multiplying herds, $45 \%$ in farrowing and farrow-to-finish herds and 50\% in finishing herds in May 1999. During 1996-1998 the estimated annual PRRS incidence was 7.5\% in nucleus and multiplying herds and $8-10 \%$ in production herds, respectively.

\section{Sweden, Finland and Norway}

Aims of the surveillance programs, target population and organisation

PRRS is a notifiable disease in Sweden, Finland, and Norway, and has never been reported in any of these countries. Each country has a surveillance program aiming to document freedom from PRRS in the nucleus and multiplying herds. In Sweden and Norway, surveillance is made also in the rest of the pig population.

In Sweden, PRRS has been screened since 1993, and since 1998 PRRS is included in the control program of the Swedish Animal Health Service. The target population in this program includes all nucleus and multiplying herds, all boars entering the semen collection centres, pig herds in Southern Sweden, as well as sows sent to slaughter. Blood samples are collected at the herds and affiliation to the program is compulsory for the breeding and multiplying herds. At slaughterhouses, blood samples are collected by the meat inspection team under the control of an official veterinarian. All samples are analysed at the National Veterinary Institute in Uppsala for antibodies to PRRS.

In Finland, PRRS was screened in the entire pig population in 1996. PRRS surveillance was included in the National Health Control Program for breeding herds in 1997. In addition, all boars entering the semen collection centres are tested. Both programs are administered by the Animal Health Authority. At slaughterhouses, blood samples are collected by the meat inspec- 
tion team under the control of the official veterinarian. Samples for antibodies are analysed at the National Veterinary and Food Research Institute in Helsinki. The boar samples are analysed for presence of antibodies to PRRS at the Danish Veterinary Institute for Virus Research, Lindholm, Denmark.

In Norway, a surveillance program for PRRS started in 1995 and all pig herds comprise the target population of the program. In addition, all boars entering the semen collection centres are tested. The program is administered by the Animal Health Authority. In pig herds, blood samples are collected by the local official veterinarians. Samples are analysed for antibodies to PRRS at the Danish Veterinary Institute for Virus Research, Lindholm, Denmark.

In each country, the surveillance is paid by the national Animal Health Authority, who is also responsible for the measures in case of interventions.

\section{Sampling}

In Swedish nucleus and multiplying herds 20 pigs are tested 1-2 times annually. The samples are collected from animals aged 5-12 months dispersed among different sections of the herd. In the Southern part of Sweden (considered to be a high risk area), 20 pigs are tested annually in 50 randomly selected production herds. In addition, 1000 randomly selected slaughtered sows from the rest of the pig herds in the country are tested annually. The total sample size varies between 5000 and 6000 samples annually.

In Finland, an annual sample size of 1300-2600 is collected randomly throughout the year from pigs that are slaughtered from progeny testing stations (no. of stations 6, total no. of test pigs 4200 in 1998). All nucleus herds send test pigs to the stations. During 1998, 1903 samples were collected from testing stations, and 690 samples from breeding boars.
In Norway, the total group of farms from which the random selection is made consists of all pig herds receiving state support (including 82 wild boar farms). Some of the largest pig herds do not receive state support, but are still included in the program.

All nucleus and multiplying herds are investigated annually, while a random sample of 290 herds is taken from the rest of the pig herds, weighted to give the highest proportion in farrowing and farrow-to-finish herds. A higher proportion of farms is selected from counties considered to be high risk areas (south-eastern and south-western part of Norway). In farrowing or farrow-to-finish herds, 10 blood samples are collected from sows, whereas 10 finishing pigs are sampled in fattening herds. In wild boar farms, 5 samples are collected from sows. The number of herds to be tested annually varies between 470 and 510, and the total sample size is $4700-5000$.

\section{Tests and testing procedures}

Samples other than boar samples are analysed for antibodies to PRRS-virus with a blockingELISA differing between the European and the US subtypes of the virus (IDEXX ${ }^{\circledR}$ Laboratories, Maine, USA). Boars are tested for PRRSantibodies with an immunoperoxidase monolayer assay (IPMA).

\section{Consequences of a positive test result}

If positive reactions are detected, the herd will immediately be closed and new blood samples from at least 20 pigs of different ages will be analyzed. In case of clinical symptoms, pigs will be euthanised and sent for necropsy. A confirmed infection with the virus will initiate an attempt to eradicated the infection by "stamping out". A restriction area with a minimum of $3 \mathrm{~km}$ radius will be determined around the infected herd. A comprehensive epizootiologic investigation will be performed to detect the 
source of the infection and the possible spread of the infection from the positive herd to neighbouring and other contact herds.

\section{Current status}

Antibodies to PRRS-virus have not been detected in any of the samples collected in Sweden, Finland, and Norway. These results, together with absence of clinical cases, support the evidence of freedom from PRRS in these countries.

\section{Iceland}

PRRS is a notifiable disease in Iceland. No clinical cases have been reported and no antibodies to PRRS-virus have been detected.

\section{Swine Enzootic Pneumonia (SEP) \\ Denmark}

Swine enzootic pneumonia, caused by $M y$ coplasma hyopneumoniae (M. hyopneumoniae), is widely prevalent in Danish swine herds. It is believed that the organism is present in all herds that are not explicit monitored for the absence of M. hyopneumoniae (SPF herds). The SPF-system is an optional program that declare presence or absence of 5-6 diseases in herds. Approximately 3000 herds participate (about $25 \%$ of total production), and approximately 1000 of these herds are free from M. hyopneumoniae. Herd health status is controlled by veterinarians 4-12 times per year and by blood sampling (yearly sampling in production herds, monthly sampling in nucleus and multiplying herds). Conventional nucleus and multiplying herds are not required to be free from $M$. hyopneumoniae, but the trade of live pigs is assisted between herds of similar infection status. Vaccination against M.hyopneumoniae is common in production herds.

\section{Sweden}

The SPF herds of the country (about $1 \%$ of the production) are controlled with respect to $M$. hyopneumoniae, and declared free from that organism. No specific monitoring program do occur within the conventional herds. Since most of the nucleus herds are known to be infected with $M$. hyopneumoniae, the organism should be regarded to be present in the production herds. The negative effects of $M$. hyopneumoniae are reduced by introducing age segregated rearing systems from birth to slaughter. During $1997,40 \%$ of the pigs reared to market weight were raised within such systems. In addition, the efficacy of vaccinations against $M$. hyopneumoniae, and definition of optimal time points for such vaccinations, are presently evaluated.

\section{Finland and Norway}

Aim of the surveillance

Both Finland and Norway have a national surveillance program for nucleus herds, and freedom of M. hyopneumoniae is a presumption for those herds to sell breeding animals. The aim of the surveillance is to keep all nucleus herds free from M. hyopneumoniae. In Norway, also multiplying herds are included in the program. In addition, in both countries there are extensive voluntary slaughter company activities aiming at certifying conventional herds free from the infection. This trend may end up to a situation where, based on national decisions, it is possible to eradicate $M$. hyopneumoniae from the entire pig population. The serological surveillance in nucleus herds started in 1992 and 1993 in Finland and Norway, respectively, and in conventional herds in 1994 and 1997, respectively.

\section{Organisation \\ Finland}

The $M$. hyopneumoniae surveillance in nucleus herds is part of the National Health Control Program for such herds. The program is admin- 
istered by the Animal Health Authority and it is compulsory for all nucleus herds. When joining the program, the herds have to pay for the analyses. Thereafter, analyses are paid by the Animal Health Authority. Colostrum samples are collected and sent to the analysis by the herd owners. All samples are analysed at the National Veterinary and Food Research Institute, Regional Laboratory in Seinäjoki. If antibodies are detected, provincial veterinarians and local official veterinarians are responsible for measures taken.

The surveillance of conventional herds is based on desicions of individual slaughter companies. Every slaughterhouse company administers it's own health control program without any formal coordination between the different programs. Basically, the programs are based on similar control strategies as the program for nucleus herds. Most slaughterhouse companies pay the costs of the analyses, when herds are joining their health control programs.

\section{Norway}

The $M$. hyopneumoniae surveillance program for nucleus and multiplying herds is based on decisions made by the Norwegian Pig Breeding Association and The Norwegian Pig Health Service. The program is compulsory, and the costs of the program are paid by a special fund based on a tax that all farmers have to pay per $\mathrm{kg}$ pork delivered to the slaughterhouse. The surveillance program for conventional herds is based on decisions made by the slaughterhouse organisations, and the costs of the program are paid by the farmers and in some areas by the slaughterhouse companies.

Both surveillance programs are administered by the Norwegian Pig Health Service. Colostrum samples are collected by the farmers. Blood samples are collected at the abattoirs. Both colostrum and blood samples are analysed at the National Veterinary Institute,
Oslo. If antibodies are detected the Norwegian Pig Health Service is responsible for measures taken.

\section{Sampling \\ Finland}

A condition of joining with the program for nucleus herds is that colostrum samples from up to 50 sows are analysed for antibodies to $M$. $h y$ opneumoniae. Thereafter, 15-25 colostrum samples are analysed biannually. A condition of joining with the programs for conventional herds is that colostrum samples from up to 30 sows are analysed. In most cases, no further sampling takes place unless clinical symptoms indicate M. hyopneumoniae infection.

\section{Norway}

The nucleus and multiplying herds were originally screened for $M$. hyopneumoniae antibodies by analyzing 60 and 40 blood samples from sows or slaughtered pigs, respectively. Biannually, 10 colostrum or blood samples from sows are collected and analysed. Conventional herds may be certified free from $M$. hyopneumoniae when a minimum of 20 colostrum samples or 20 blood samples from slaughtered finishing pigs are analysed and no antibodies to $M$. hyopneumoniae are detected. Thereafter, 10 colostrum or blood samples are analysed once a year.

\section{Consequences of a positive result}

Both serum and colostrum samples are analyzed for the presence of antibodies to $M$. hyopneumoniae by a monoclonal blocking ELISA (Mycoplasma hyopneumoniae ELISA ${ }^{\circledR}$, DAKO A/S, Glostrup, Denmark). If an nucleus or multiplying herd tests positive, confirmatory tests will take place. If the infection is confirmed, the herd can not sell breeding animals any more. An eradication program will take place after the acute phase of the infection. In Finland, such a 
program is supported economically by the Animal Health Authority. If a conventional herd looses it's M. hyopneumoniae free status, negative economical consequences will take place. The price of pigs delivered for fattening is reduced by $7-10 \%$ and $4 \%$ in Finland and Norway, respectively.

\section{Certification}

Both in Finland and Norway, all nucleus herds (and multiplying herds in Norway) are certified free from $M$. hyopneumoniae, if the requirements of the surveillance programs are met, clinical symptoms indicating SEP are not detected during the quarterly veterinary visits and the prevalence of pneumonia recorded at meat inspection remains low.

\section{Regulated control of disease spread}

In Finland, the nucleus herds are allowed to buy breeding animals from other similar certified herds. At such occasions, great attention has to be paid on common practises for the prevention of possible disease spread. In Norway, the nucleus herds have been totally closed for several years. Multiplying herds are allowed to buy purebred sows from only one single certified nucleus herd.

\section{Current status}

\section{Finland}

In 1995, all nucleus herds were screened and 4 herds were concluded to be infected. Since then, one herd has been reinfected annually. The surveillance in the nucleus herds during 1996 to 1998 is shown in table 4. During 1991, the prevalence of sow herds with antibodies to M. hyopneumoniae was $30 \%$ and $8 \%$ in Western and in Southwestern Finland, respectively. Since then the number of colostrum and blood samples collected from conventional farrowing herds has been steadily increasing. Today, at least one half of the total of 2300 conventional
Table 4. Summary of the Mycoplasma hyopneumoniae surveillance in Finnish nucleus pig herds $(n=150)$ during 1996-1998 (number of samples with antibodies in blocking-ELISA / total number of samples).

\begin{tabular}{lccc}
\hline & 1996 & 1997 & 1998 \\
\hline $\begin{array}{l}\text { Colostrum } \\
\text { samples }\end{array}$ & $49^{\mathrm{a}} / 4958$ & $22^{\mathrm{b}} / 5427$ & $15^{\mathrm{d}} / 3808$ \\
$\begin{array}{l}\text { Confirmatory } \\
\text { blood samples }\end{array}$ & $0 / 119$ & $18 \mathrm{c} / 298$ & $17 \mathrm{e} / 345$ \\
\hline
\end{tabular}

a 44 positive samples from 1 herd; other positive samples single positives in a herd

b 7 positive samples from 1 herd; other positive samples single positives in a herd

c all positive samples from 1 herd

d 8 positive samples from 1 herd; other positive samples single positives in a herd

e all positive samples from 1 herd

farrowing herds are certified by the slaughterhouse companies as $M$. hyopneumoniae free herds.

\section{Norway}

All nucleus and multiplying herds were screened during 1994-1996 (table 5.) Eleven of the total of 189 herds were concluded to be infected. Since then, 2 nucleus and 3 multiplying herds have been reinfected. Two of the multiplying herds were infected by breeding sows bought from one of the infected nucleus herds. Preliminary studies in about 700 conventional herds indicate that the prevalence of farrowing and farrow-to-finish herds infected with $M$. hyopneumoniae varies between 5 and $40 \%$ in different counties of Norway.

\section{Iceland}

SEP is present in the majority of Icelandic pig herds.

\section{Progressive Atrophic Rhinitis (PAR)}

In Denmark, toxin producing strains of $\mathrm{Pa}$ steurella multocida, the causative agent of progressive atrophic rhinitis, are monitored twice 
Table 5. Summary of the screening of Norwegian nucleus and multiplying pig herds for Antibodies in blocking ELISA to Mycoplasma hyopneumoniae in blood samples during 1994-1996.

\begin{tabular}{lccc}
\hline & $\begin{array}{c}\text { Total no. of } \\
\text { samples }\end{array}$ & $\begin{array}{c}\text { No. of samples } \\
\text { with antibodies (\%) }\end{array}$ & $\begin{array}{c}\text { No. of herds with a } \\
\text { confirmed infection (\%) }\end{array}$ \\
\hline Elite breeding herds $(\mathrm{n}=110)$ & 7101 & $177(2.5)$ & $5(4.5)$ \\
Multiplying herds $(\mathrm{n}=79)$ & 3078 & $156(5.1)$ & $6(7.6)$ \\
\hline
\end{tabular}

every winter season in nucleus and multiplying herds. These herds are defined free of the disease. One herd test is based on collection of 20 nasal swab samples pooled 2 and 2 in 10 veils. Samples are analysed for $P$. multocida toxin (Pasteurella multocida toxin ELISA ${ }^{\circledR}$, DAKO A/S, Glostrup, Denmark).

In Sweden, toxin producing strains of P. multocida were eradicated from the nucleus herds during 1993-94. The nucleus and multiplying herds are defined free from infections with toxin producing strains of $P$. multocida since 1995 , which also is proven by annual control. Between 5000 and 8000 nasal swab samples are analysed annually for P. multocida toxin by ELISA.

\section{References}

Anonymous: Animal Diseases and Animal Welfare in Finland in 1998. Finnish Ministry of Agriculture and Forestry. Veterinary and Food Department. Helsinki, Finland. 1999.

Anonymous: Zoonoses in Finland in 1995-1997. Finnish Ministry of Agriculture and Forestry. Veterinary and Food Department. Helsinki, Finland. 1999.

Anonymous: The Finnish Salmonella Control Programmes for Live Animals, Eggs and Meat. Finnish Ministry of Agriculture and Forestry. Veterinary and Food Department. Helsinki, Finland. 1994.

Elvander $M$, Larsson B, Engvall A, Björnerot L, Klingeborn B, Gunnarsson A: Nationwide surveys of TGE/PRCV, CFS, PRRS, SVD, $L$. pomona, and B. suis in pigs in Sweden. 7th Proc. Symp. Int. Epid. Econ. Vet. 07.B39. 1997.

Mousing J, Jensen PT, Halgaard C, Bager F, Feld N,
Nielsen B, Nielsen JP, Bech-Nielsen S: Nationwide Salmonella enterica surveillance and control in Danish slaughter swine herds. Prev. Vet. Med. 1997, 29, 247-261.

Nielsen B: Update on Danish Salmonella reduction program. Proceedings of the 6th annual swine disease conference for swine practitioners, Ames, Iowa, USA, November 12-13, 1998. pp. 149-152.

Rautiainen E: The prevalence of Mycoplasma hyopneumoniae in pig herds in Western Finland based on the demonstration of antibodies in colostrum by ELISA. Acta Vet. Scand. 1998, 39, 325-330.

Robertsson JA, Holmström A, Björnerot L, Lindqvist $H$ : Control of PRRS in Sweden. Proceedings of the PRRS and Aujeszky Disease congress, Ploufragan, France, June 21-24, 1999. pp. 305306.

Tuovinen V, Levonen K, Gröhn Y, Straw B: The prevalence of some economically

important swine diseases in farrowing units in Southwestern Finland. Prev.Vet.Med. 1994, 19, 85-100.

Wahlström H, Bergström K, Engvall A, Gunnarsson $A$, Lindqvist $H$, Berge $C$, Wierup M: The Swedish Salmonella control of pig and pork production. Proceedings of the 15th IPVS congress, Birmingham, England, 5-9 July, 1998. p. 73.

Wallgren P, Mattsson S, Stampe M, Molander B, Lindblad M, Wierup M: Control of infections with toxin-producing Pasteurella multocida in herds affected with atrophic rhinitis. Proceedings of the 13th IPVS congress, Bangkok, Thailand, 26-30 June, 1994. p.123.

Wierup M: Status för kontrollprogrammet: Nyssjuka hos svin, progressiv atrofisk rhinit. (Status of the control program against progressive atrophic rhinitis). Swedish Annu. Vet. Congress 1995. pp. 185-189. 